Images: Acute urinary retention due to urethral steinstrasse

\title{
Images - Acute urinary retention due to urethral steinstrasse
}

\author{
Andrew Morton ${ }^{1,2}$; Lachlan Gordon ${ }^{2}$; Ross Fowler ${ }^{2}$; Rachel Esler ${ }^{2}$; Nigel Dunglison ${ }^{2}$; \\ Matthew J. Roberts ${ }^{2,3}$ \\ ${ }^{1}$ Faculty of Medicine, The University of Queensland, Brisbane, Queensland, Australia; ${ }^{2}$ Department of Urology, \\ Royal Brisbane and Women's Hospital, Queensland, Australia; ${ }^{3}$ The University of Queensland Centre for \\ Clinical Research, Herston, Australia
}

Cite as: Morton A, Gordon L, Fowler R, et al. Images - Acute urinary retention due to urethral steinstrasse. Can Urol Assoc J 2020 October 27; Epub ahead of print.

http://dx.doi.org/10.5489/cuaj.6725

Published online October 27, 2020

$* * *$

\section{Introduction}

Steinstrasse, or "stone street", of the ureter is a well-documented complication following extracorporeal shock wave lithotripsy where incomplete fragmentation leads to obstruction and accumulation of multiple calculi ${ }^{1,2}$. In contrast, steinstrasse of the urethra is exceedingly rare; we present a case causing acute urinary retention some weeks following cystolitholapaxy for bladder calculi.

\section{Case presentation}

A 70 year-old man presented to the emergency department with suprapubic pain and urinary retention. Examination revealed normal vital signs, a palpable bladder, and penile crepitus with a small stone visible at the external urethral meatus. Blood investigations were unremarkable, showing normal renal function (serum creatinine $72 \mathrm{umol} / \mathrm{L}$ ), white cell count $\left(8.8 \times 10^{9}\right)$, and electrolytes. Urinalysis demonstrated raised leucocytes $\left(>500 \times 10^{6} / \mathrm{L}\right)$, erythrocytes $\left(>500 \times 10^{6} / \mathrm{L}\right)$, and mixed skin flora on culture. Plain imaging (Figure 1) revealed multiple calculi along the length of the urethra, confirming the diagnosis of urethral steinstrasse.

Further history revealed that the patient had undergone an elective combined transurethral resection of prostate (TURP) and cystolitholapaxy for symptomatic benign prostatic hyperplasia and multiple $2 \mathrm{~cm}$ bladder calculi three weeks prior to presentation. Following meatotomy for submeatal stenosis, initial calculus fragmentation using a lithotrite failed due to hard calculi composition. Without an option for open cystolithotomy or high power surgical laser, further prolonged pneumatic lithotripsy was performed followed by TURP. After evacuation of prostate chips and bladder calculi, remaining limited stone fragments (estimated $<5 \mathrm{~mm}$ ) were thought to be small enough to pass spontaneously. The patient underwent uncomplicated routine TURP care including a successful trial-of-void and discharge on the second post-operative day. 
After evaluation in our emergency department, the patient underwent emergent endoscopic management with further meatotomy, and completion cystolitholapaxy. The $5 \mathrm{~mm}$ obstructing stone was removed with forceps under direct vision. Further stone fragments within the distal urethra were removed using an endoscopic grasper, whereas proximal stones were manipulated back into the bladder for further fragmentation. On the first postoperative day, the urinary catheter was successfully removed and the patient was discharged. Stone analysis revealed calcium oxalate composition. The patient remained well and symptom-free 3 months postoperatively.

\section{Conclusions}

Urethral steinstrasse has previously been reported following ESWL for large renal staghorn calculi $^{3}$, in the post-cystolitholapaxy setting ${ }^{4,5}$, and also in two patients without any history of stone disease $\mathrm{e}^{6,7}$. In this case, bladder calculi occurred as a complication of BPH which are most commonly managed endoscopically ${ }^{8-10}$. The initial prolonged, difficult cysttolitholopaxy and concomitant submeatal stenosis lead to failure of spontaneous passage of remaining stone fragments resulting in obstruction and steinstrasse. This highlights the importance of careful stone evacuation at cystolitholapaxy, particularly in the setting of known urethral obstruction, as the most distal obstructing stone fragment can be as small as $5 \mathrm{~mm}$. Whilst minimally invasive endoscopic techniques are usually preferred, patients should be adequately counselled for open cystolithotomy when required. Urethral steinstrasse is a rare occurrence that may arise following cystolitholapaxy, and patients should be monitored for this possible complication.

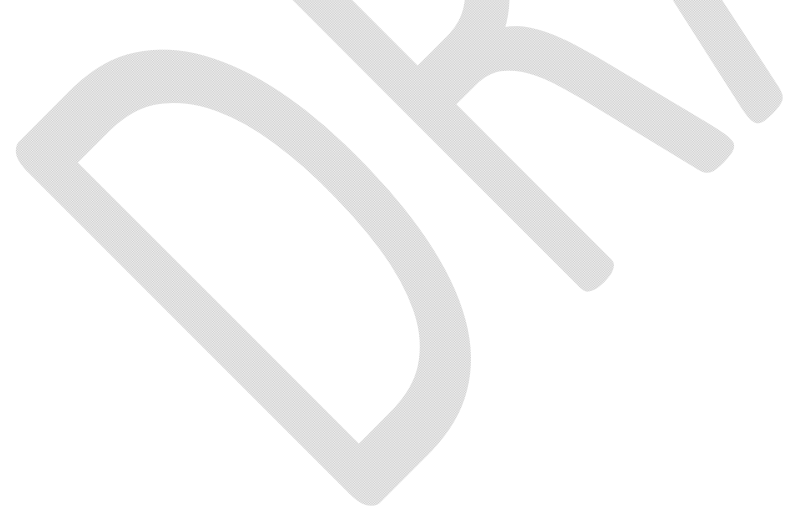




\section{References}

1. Kim SC, Oh CH, Moon YT et al. Treatment of steinstrasse with repeat extracorporeal shock wave lithotripsy: experience with piezoelectric lithotriptor. J Urol. 1991;145:489-91.

2. Ordon M, Andonian S, Blew B et al. CUA Guideline: Management of ureteral calculi. Can Urol Assoc J. 2015;9:E837-51.

3. Biyani CS, Bhatia V, Baliga D. Urethral steinstrasse--clinical experience and radiographic findings. Clin Radiol. 1993;48:273-4.

4. Atikeler MK, Yuzgec V, Gecit I et al. Urethral stone street. Int Urol Nephrol. 2005;37:315.

5. Tsing VLM, Mansbridge MM, Latif ER et al. Unusual case of urethral steinstrasse following laser cystolitholapaxy. BMJ Case Rep. 2017;2017.

6. Adebayo SA, Chibuzo INC, Takure AO et al. Urethral steinstrasse: Day case care. Clin Case Rep. 2018;6:1697-700.

7. Vaddi SP, Devraj R, Reddy V et al. Urethral steinstrasse causing acute urinary retention. Urology. 2011;77:594-5.

8. Jiwrajka M, Yaxley W, Perera M et al. Review and update of benign prostatic hyperplasia in general practice. Aust J Gen Pract. 2018;47:471-5.

9. Morton A, Williams M, Perera $\mathrm{M}$ et al. Management of benign prostatic hyperplasia in the 21 st century: temporal trends in Australian population-based data BJU Int. 2020; In press:In press.

10. Nickel JC, Aaron L, Barkin J et al. Canadian Urological Association guideline on male lower urinary tract symptoms/benign prostatic hyperplasia (MLUTS/BPH): 2018 update. Can Urol Assoc J. 2018;12:303-12.

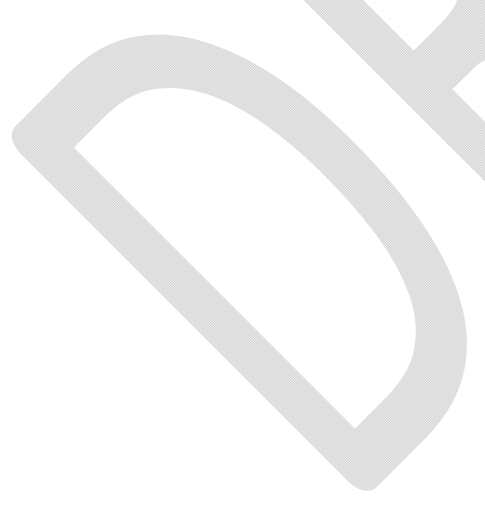




\section{Figures and Tables}

Fig. 1. Plain X-ray of the pelvis showing multiple urolithiasis within the urethra (arrow). (A) Anterior-posterior view. (B) Oblique view.

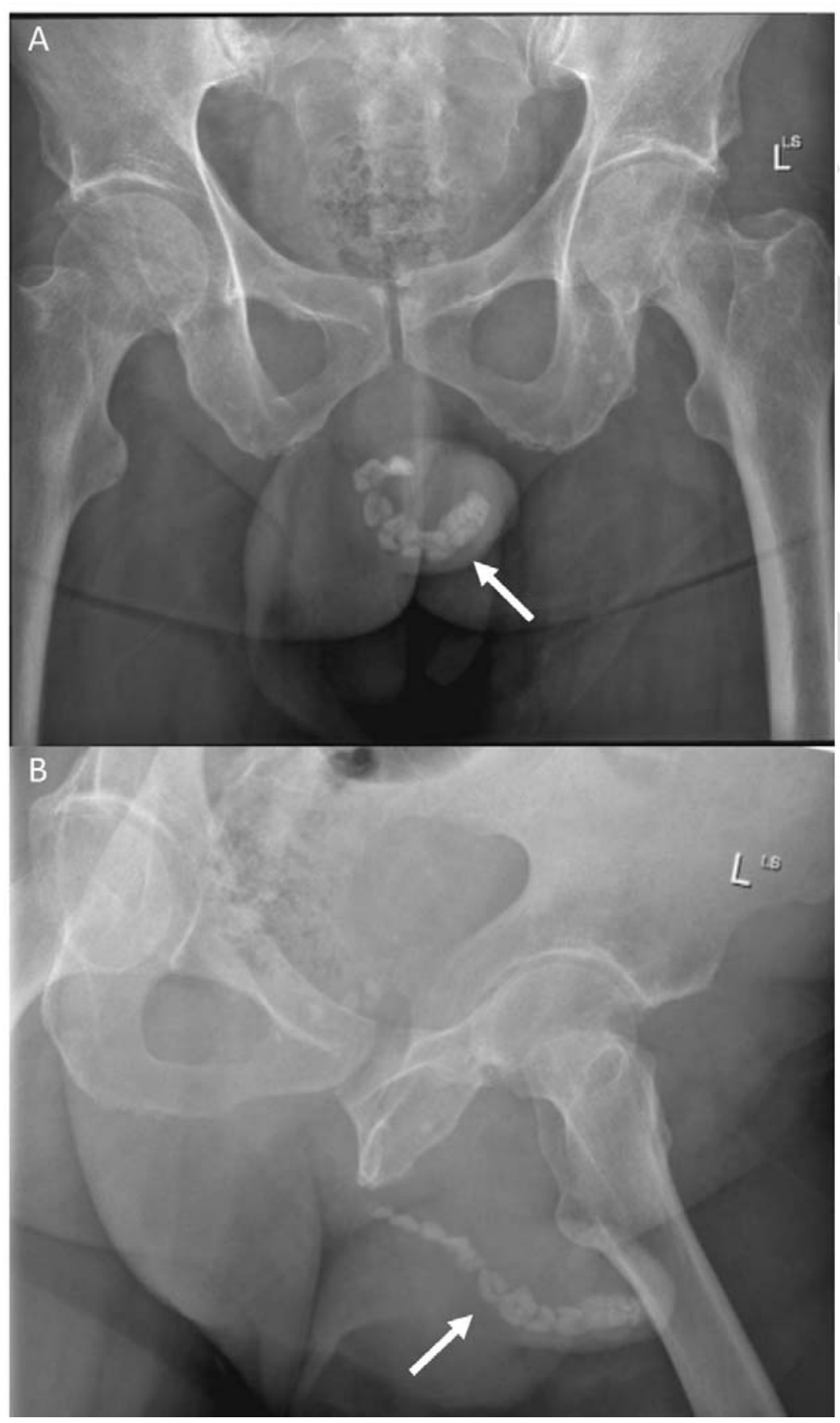

